\title{
Formation of Nonwoven Webs with Electrostatic Field Effect
}

\author{
Ahmad Kanah ${ }^{1 *}$, Dr. Mohamed Turkawi ${ }^{2}$
}

\section{Authors affiliations: \\ $1 *$ ) Department of Textile Engineering - College of Chemical and Petroleum Engineering - Al-Baath University, Homs, Syria. ahmad.kanah94@gmail.com}

2) Department of Textile Engineering - College of Chemical and Petroleum Engineering - Al-Baath University, Homs, Syria. mturkawi@hotmail.com

\section{Paper History:}

Received: $22^{\text {th }}$ Oct. 2019

Revised: 6 ${ }^{\text {st }}$ Dec. 2019

Accepted: 29th Jan. 2020:

\begin{abstract}
:
The non-woven materials industry is one of the fastest-growing industries in the world with the ability to produce materials in less time, specifications, and better prices. nonwoven materials are defined as a web of guided or random fibers that are bonded by friction, interlacement or adhesion. In this research, the rotary electrospinning system was used and a prototype was made to study the process and the complete visualization in terms of the correlation of the electrostatic forces to the formation of nanofibers by preparing polymeric solutions and exposing them to the electric field between the positive electrode (the serrated cylinder) and the Grounded electrode (plate) and produced high-precision fibers with a diameter $(185 \mathrm{~nm})$ at $25 \mathrm{kV}$, whereas the installation of polyvinyl alcohol (PVA) was with different concentrations and the formed fibers possessed an effective surface and deposited on a collector electrode forming nonwoven webs and high productivity is the most important feature of this system compared with the traditional electrospinning system.
\end{abstract}

Keywords: Nanospider, Rotary Electrospinning, Non-Woven, Nanofibers.

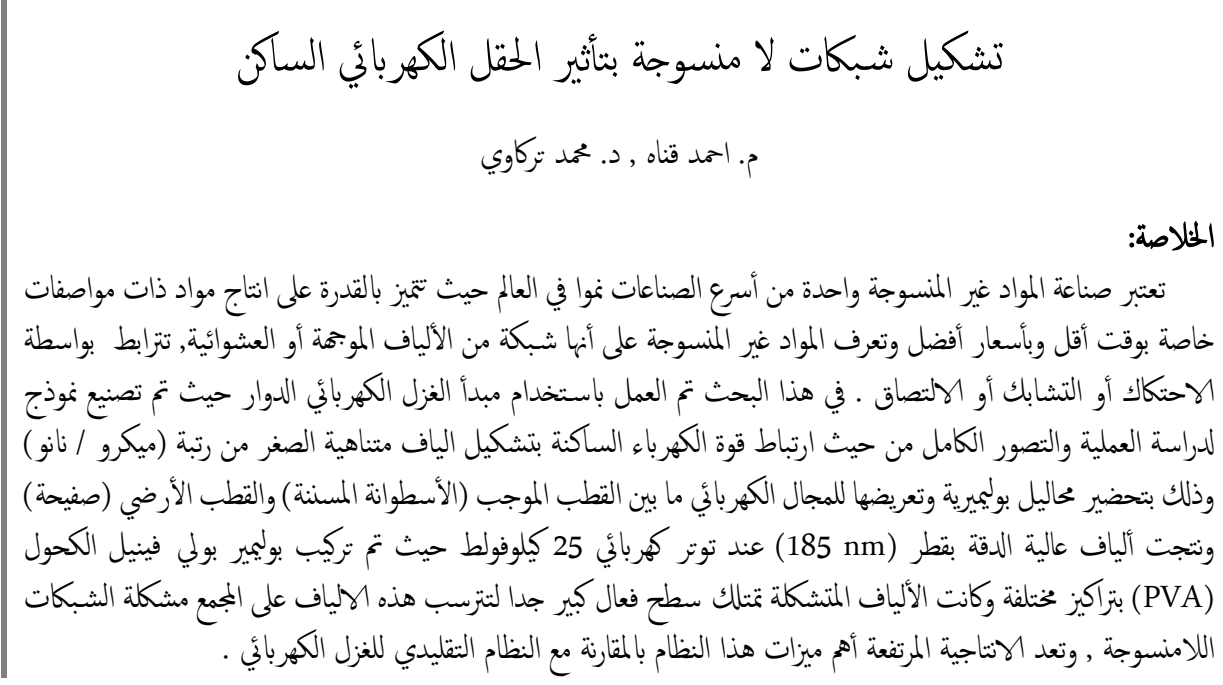

\section{Introduction}

Non-woven fabrics are used today in all areas of the life, where they are used in the industrial field, such as filters, cable's' insulators, CFRP, packaging, and also home furniture, such as fillings, walls' covers, curtains and floors, as well as, transportation, seeds' covering and herb protection. One of the most important reasons for its rapid development is it can be used in the Medical field as, vascular grafting, tissue engineering, and orthopedic surgery.

The process starts from the polymer solution which is stable as droplets at the top of the spinning cylinder, after a certain threshold the electric field overcomes the surface tension forces, that the droplets go under a quick elongation mechanism in the region between the head and spinning complex, so that how the fiber is been allowed to be formed to become long and very thin. On the way to the compound, the solvent evaporates from the polymer solution and
Solid fibers are placed on the compound at high speeds (49 $\mathbf{m s}^{-1}$ or more) to form the non-woven networks as shown in Figure (1).

\section{1-1 Effective Factors On Electrospinning: 1-1-1 Factors related to the solution:} \section{Concentration:}

The concentration of the polymeric solution plays an important role in the formation of fibers using the electrospinning method. Four bottom-up concentrations can be observed:

- When the concentration is too low, we will get nanoparticles within the polymer solution, then an electrical atomization occurs instead of the electrospinning due to low viscosity and high surface tension of the solution [2].

- When the concentration is slightly higher, we will get a mixture of spray and fiber $[3,4,5]$.

NJES is an open access Journal with ISSN 2521-9154 and eISSN 2521-9162

This work is licensed under a Creative Commons Attribution-NonCommercial 4.0 International License 
- When the concentration is appropriate, we will get good nanofibers [3,4,5].

- If the concentration is too high, no nanofibers will be formed and we will get spiral-shaped microfibers [6].

Usually, the concentration of the solution used in the electrospinning is low so that the electrostatic force can pull the fibers toward the collector, but it shouldn't decrease and the value of this concentration doesn't rise above a certain limit.

\section{Molecular Weight:}

At a given concentration molecular weight reduction leads to the formation of nodes instead of fibers, and the increased molecular weight leads to an increased fiber formation $[7,8,9]$. the electrospinning of polymers that have a high molecular weight is easier because the high molecular weight facilitates the process of controlling viscosity, but this doesn't mean that the high molecular weight of the polymer is a necessary condition for the possibility of spinning it electrically, but the basic condition is that the value of the molecular weight be sufficient to achieve a correlation between the molecule chains Polymeric.

\section{Viscosity of solution:}

The viscosity of the solution can be adjusted by controlling its concentration where it should be noted that the viscosity, concentration of the solution, and molecular weight are related factors [8]. experimentally, it was found that nanofibers are obtained at low viscosity values, but this is associated with superficial defects, which are beads, and at high viscosity, fibers are obtained with high diameters.

\section{1-2 Technical factors:}

\section{Applicable voltage:}

Electrospinning is the only technique that uses electrostatic force to produce nanofibers from solutions or molten polymers of microbial or nanoscale diameters and with a very high effective surface.

The Taylor cone is formed only at a threshold value of the voltage (critical voltage). Studies have shown that high electric fields affect the formation of fibers with larger diameters when fixing the distance [10].

When applying the critical voltage, the electrostatic force (force columbic) pulls the polymeric jets and the polymeric thread forms(filament), and as a result of the traction force, it moves very quickly towards the collector, causing elongation and a large decrease in its diameter, then it turns into nanofibers or micro-fibers that are placed on the surface of the collector after To losing its charge from the metal head of the electrode.

\section{1-3 Surroundings Factors:}

Both temperature and humidity affect the morphological and physical characteristics of nanofibers, where temperature increase leads to the formation of fibers with a smaller diameter, on the other hand, low humidity causes an increase of the speed of the evaporation of the solvent and leads to dry it completely. On the contrary high humidity leads to the formation of fibers with larger diameters because the charges on the fiber can be modified, and the tensile strength applied to the fiber becomes smaller [11-12].

\section{The objective of the research:}

The research aims to produce high-precision webs of polymers dissolved in water, acids, or solvents, as well as work to improve their structure and morphology by using a device that using a rotary electrospinning technique (needleless), also, to use them in the industrial field.

\section{Experimental}

\section{3-1 Materials}

The dimensions of the device is $50 \times 30 \times 30 \mathrm{~cm}$

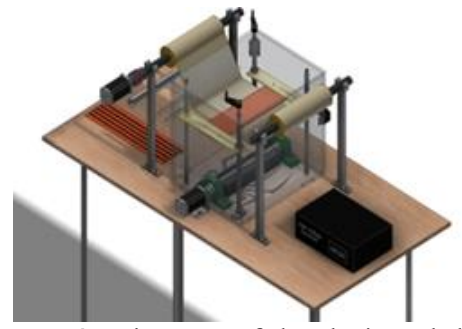

Figure 1: Diagram of the designed device

The device shown in Figure (1) consists of an outer box containing a spinning cylinder inside a Tank made of Plexiglass (insulating material) in addition to high voltage (HV), motor, motor bed, and collector which is a metal plate that collects nanofibers [13] The collector consists of different materials (chrome, stainless steel).

The device has many advantages:

The designed device has several spinning heads, each of which operates on its own.

The designer device has several Forms for the collector and different materials and sizes

Reducing the electrostatic force needed to start producing nanofibers.

The transition from a low productivity of the traditional electrospinning system to very high productivity

- Eliminate the problem of clogging of the extruder in the traditional electrospinning

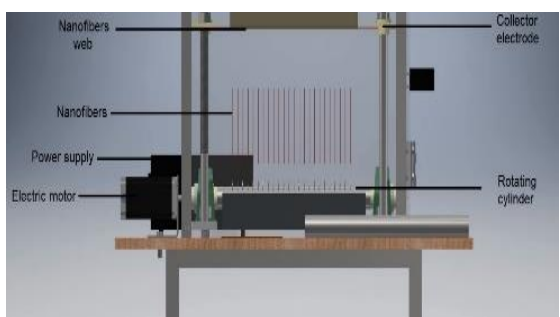

Figure 2: Work principle of the designed device

The device works according to the rotary electrospinning system, as shown in Figure 2. It starts when the serrated cylinder is exposed to the positive electrical voltage and thus the drops placed on the heads "which are transferred from the bottom surface of the cylinder inside the tank to the upper surface" begin to gain positive charges and after a certain charge time it collapses The strength of the surface tension of these droplets, and thus the Taylor cone begins to 
formation, Figure (3) to form nanofibers, which in turn deposited on the collector forming nanoscale webs.
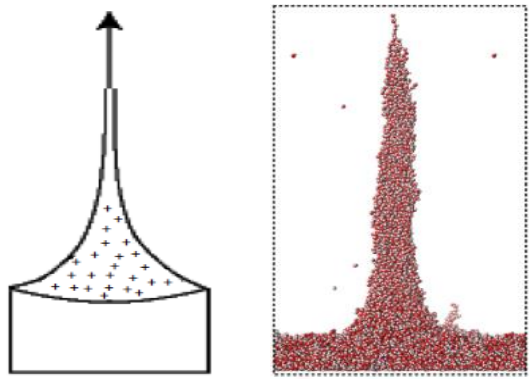

Figure 3: Taylor cone

\section{3-2 Measurements}

In this paper, polyvinyl alcohol polymer was Prepared in concentrations $(6 \%-12 \%)$. Figure (4).

\section{Experiment 1:}

The polymeric solution was used at a concentration of $(12 \%)$ where the liquid was inside the tank in the device shown in Figure (1) and the collector was placed at a distance of $(8 \mathrm{~cm})$ from the spinning drum. A stainless-steel plate was used to collect the fibers, and a voltage up to $(40 \mathrm{kV})$ was applied by using the high voltage transformer which is located in the high voltage laboratory at Damascus University Faculty of Mechanical and Electrical Engineering Electrical Power Department. Table (1) shows the parameters of the experiment. The results indicate that the fibers were not formed due to the high viscosity of the liquid where the static electricity of the field was unable to overcome the surface tension of the liquid.
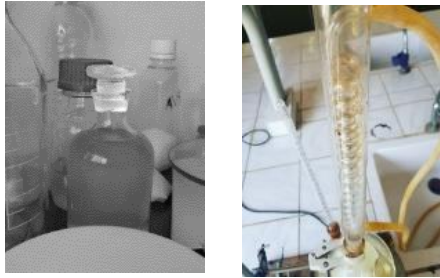

Figure 4: Preparation of polymeric solution Table 1: Process parameters of the roller electrospinning.

\begin{tabular}{|c|c|c|c|c|c|}
\hline 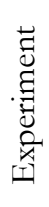 & 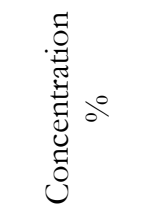 & 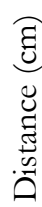 & $\begin{array}{l}\Xi \\
\Xi \\
0 \\
50 \\
\frac{\pi}{0} \\
>0\end{array}$ & 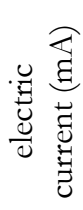 & 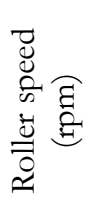 \\
\hline 1 & $12 \%$ PVA & 8 & $20-40$ & $\mathrm{AC}$ & 3 \\
\hline 2 & $6 \%$ PVA & 8 & 20 & $\mathrm{AC}$ & 1.25 \\
\hline 3 & $6 \%$ PVA & 8 & 25 & $\mathrm{AC}$ & 1.25 \\
\hline 4 & $6 \%$ PVA & 8 & 30 & Ac & 1.25 \\
\hline
\end{tabular}

\section{Experiment 2:}

The polymeric solution was used at a concentration of $(6 \%)$ and the stainless- steel plate was used as a collector.
The results showed that the fibers were not observed with the naked eye, which lead to form a nonwoven web's that settled on the collector where the sample was imaged using a scanning electronic microscope (SEM) located within the Atomic Energy Commission and the average fibers diameters were (202nm) as shown in Figure (5).

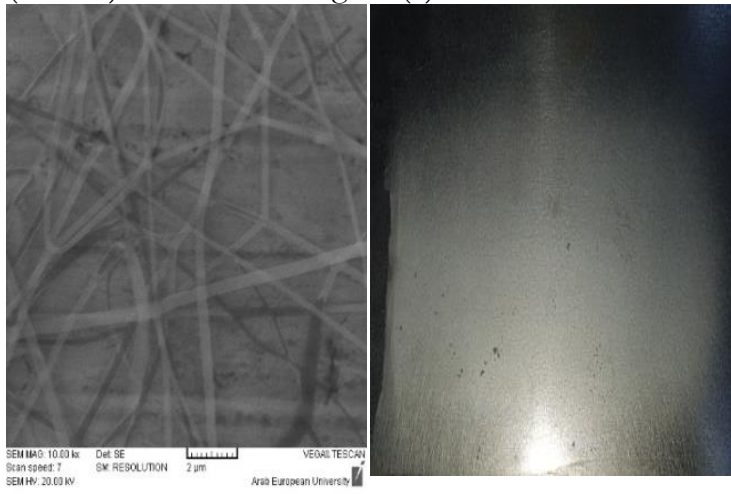

Figure 5: The SEM images of $6 \%$ PVA nonwoven nanofibers

\section{Experiment 3:}

The polymeric solution was used at a concentration of $(6 \%)$ and the chrome plate was used as a collector. The results showed that the fibers were settled on the collector where the Chrome metal formed the fibers faster than the stainless steel and the average diameters $(185 \mathrm{~nm})$ as shown in Figure (6).

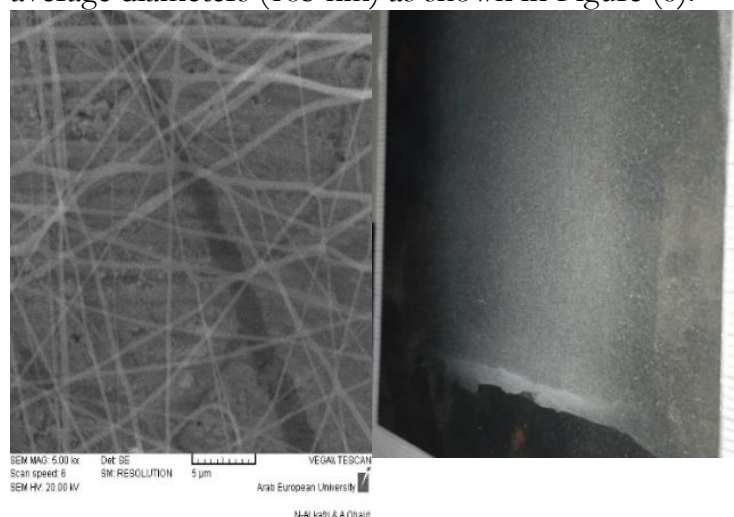

Figure 6: The SEM images of $6 \%$ PVA nonwoven nanofibers $(25 \mathrm{KV})$.

\section{Experiment 4:}

The parameters of experiment 3 were used similarly in this experiment with an increase in voltage. the results showed high-precision fibers stabilized on the collector more regularly with average diameters $(193 \mathrm{~nm})$ as shown in Figure (7). 

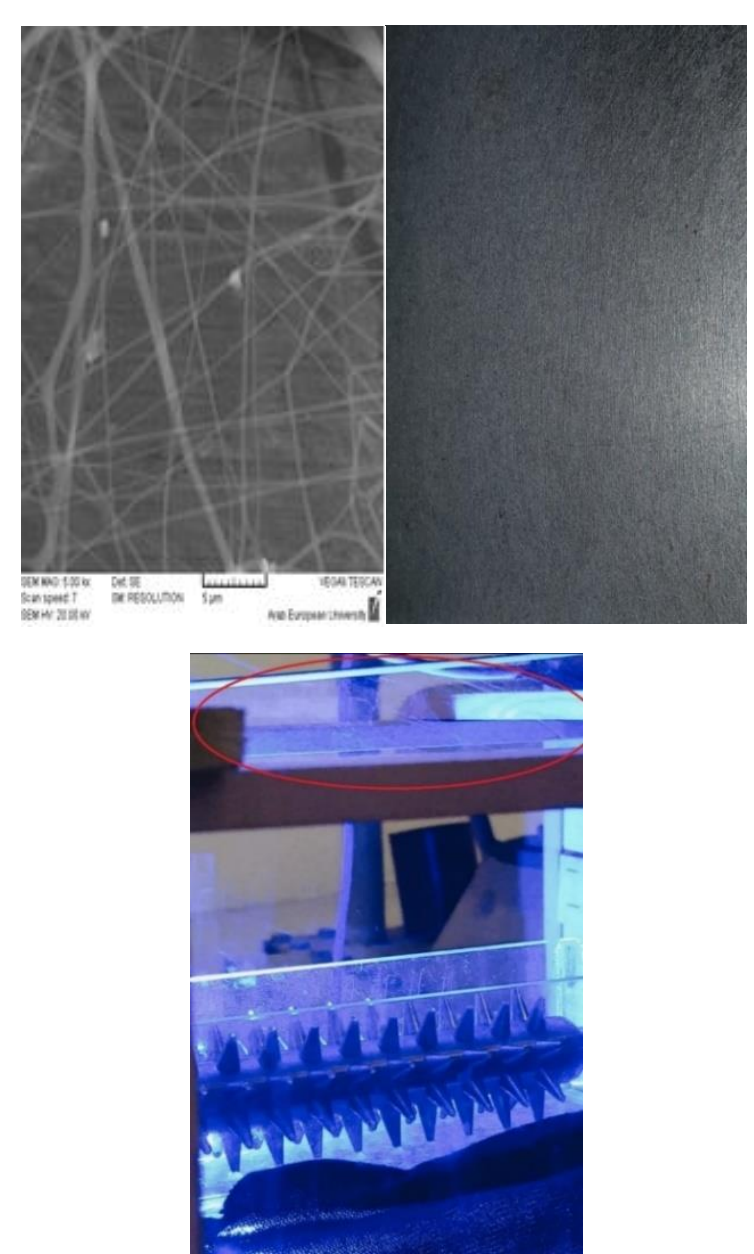

Figure 7: SEM images of the PVA nanofibers electrospun by cylinder nozzles.

\section{4- Conclusions:}

The nanofibers were spun using a polyvinyl alcohol polymer, and the need for precision in preparation is praised which is highly reflects on the diameter of the fibers and their spin ability. Adjusting the concentration, polymerization, viscosity, and density have a great role in overcoming the surface tension strength and thus starting the production process. on the other hand, the surface tension is of greater value when the liquid layer is coherent, and therefore we need high values of electrostatic force $(50-80 \mathrm{KV})$ to produce fibers, and from here it has been working to reduce electric field to reach $(20-35 \mathrm{KV})$ by finding A solution that overcomes the strength of the surface tension of the fluid level by designing a serrated cylinder and thus converting the surface of the liquid into drops located on the heads of the serrated cylinder and thus not requiring a high voltage to break the strength of the surface tension of the polymeric fluid layer.

In comparison with the traditional electrospinning system (needle - cylinder) which is about $(0.1 \mathrm{~g} / \mathrm{h})$, the productivity of the manufactured device (cylinder plate) was about $(1.25 \mathrm{~g} / \mathrm{h})$ for the same parameters.

It is noted from experiments that the increase in the strength of electrical tension leads to improved fiber morphology and increase density.
When the viscosity is high, the fibers will not be formed and therefore the non-woven webs will not be formed also as in experiment (1).

When the viscosity is appropriate, non-woven webs will be formed at a suitable voltage $(20 \mathrm{kV})$ where the average diameters of the nanofibers $(202 \mathrm{~nm})$ as in experiment (2).

The fibers began to form when the voltage of $20 \mathrm{kv}$, and reached the best uniformity at the voltage of $30 \mathrm{kv}$ as in experiment (4) where the average diameters of the nanofibers (193 nm).

Finally, it was experimentally found that whenever the collector's volume is relatively greeter is better for the fibers' formation speed.

\section{5-References}

[1] Greiner, A., \& Wendorff, J. H., Electrospinning: a fascinating method for the preparation of ultrathin fibers. Angewandte Chemie International Edition, 46(30), 5670-5703, 2007.

[2] Deitzel, J. M., Kleinmeyer, J., Harris, D. E. A., \& Tan, N. B., The effect of processing variables on the morphology of electrospun nanofibers and textiles. Polymer, 42(1), 261-272, 2001.

[3] Eda, G., \& Shivkumar, S., Bead-to-fiber transition in electrospun polystyrene. Journal of Applied Polymer Science, 106(1), 475-487, 2007.

[4] Fong, H., Chun, I., \& Reneker, D. H., Beaded nanofibers formed during electrospinning. Polymer, 40(16), 4585-4592, 1999.

[5] Lee, K. H., Kim, H. Y., Bang, H. J., Jung, Y. H., \& Lee, S. G., The change of bead morphology formed on electrospun polystyrene fibers. Polymer, 44(14), 4029-4034, 2003.

[6] Yang, Q., Li, Z., Hong, Y., Zhao, Y., Qiu, S., Wang, C. E., \& Wei, Y., Influence of solvents on the formation of ultrathin uniform poly (vinyl pyrrolidone) nanofibers with electrospinning. Journal of Polymer Science Part B: Polymer Physics, 42(20), 3721-3726, 2004.

[7] Koski, A., Yim, K., \& Shivkumar, S., Effect of molecular weight on fibrous PVA produced by electrospinning. Materials Letters, 58(3-4), 493497, 2004.

[8] Cengiz, F., Dao, T. A., \& Jirsak, O., Influence of solution properties on the roller electrospinning of poly (vinyl alcohol). Polymer Engineering \& Science, 50(5), 936-943, 2010.

[9] Zhao, Y. Y., Yang, Q. B., Lu, X. F., Wang, C., \& Wei, Y., Study on correlation of morphology of electrospun products of polyacrylamide with ultrahigh molecular weight. Journal of Polymer Science Part B: Polymer Physics, 43(16), 2190-2195, 2005.

[10] Zhang, C., Yuan, X., Wu, L., Han, Y., \& Sheng, J., Study on morphology of electrospun poly (vinyl alcohol) mats. European polymer journal, 41(3), 423-432, 2005.

[11] Mit-uppatham, C., Nithitanakul, M., \& Supaphol, P., Ultrafine electrospun polyamide-6 fibers: effect of solution conditions on morphology and average fiber diameter. Macromolecular Chemistry and Physics, 205(17), 2327-2338, 2004. 
[12] Casper, C. L., Stephens, J. S., Tassi, N. G., Chase, D. B., \& Rabolt, J. F., Controlling surface morphology of electrospun polystyrene fibers: effect of humidity and molecular weight in the electrospinning process. Macromolecules, 37(2), 573-578, 2004.

[13] Jirsak, O., Sanetrnik, F., Lukas, D., Kotek, V., Martinova, L., \& Chaloupek, J., U.S. Patent No. 7,585,437. Washington, DC: U.S. Patent and Trademark Office, 2009.

[14] Migliaresi, C., Ruffo, G. A., Volpato, F. Z., \& Zeni, D., Advanced electrospinning setups and special fibre and mesh morphologies. Electrospinning for advanced biomedical applications and therapies. Smithersrapra, United Kingdom, 23-68, 2012.

[15] Liu, Y., \& He, J. H., Bubble electrospinning for mass production of nanofibers. International Journal of Nonlinear Sciences and Numerical Simulation, 8(3), 393-396, 2007.

[16] Lukas, D., Sarkar, A., \& Pokorny, P., Selforganization of jets in electrospinning from free liquid surface: A generalized approach. Journal of Applied Physics, 103(8), 084309, 2008.

[17] Yarin, A. L., \& Zussman, E., Upward needleless electrospinning of multiple nanofibers. Polymer, 45(9), 2977-2980, 2004.

[18] Zhang, Y., Zhang, L., Cheng, L., Qin, Y., Li, Y., Yang, W., \& Li, H., Efficient preparation of polymer nanofibers by needle roller electrospinning with low threshold voltage. Polymer Engineering \& Science, 59(4), 745751, 2019.

[19] Yalcinkaya, F., Yalcinkaya, B., \& Jirsak, O. Analysis of the effects of rotating roller speed on a roller electrospinning system. Textile Research Journal, 87(8), 913-928, (2017).

[20] El-Newehy, M. H., Al-Deyab, S. S., Kenawy, E. R., \& Abdel-Megeed, A. Nanospider technology for the production of nylon-6 nanofibers for biomedical applications. Journal of Nanomaterials, 2011, 9, (2011).

[21] Yu, M., Dong, R. H., Yan, X., Yu, G. F., You, M. H., Ning, X., \& Long, Y. Z. Recent advances in needleless electrospinning of ultrathin fibers: from academia to industrial production. Macromolecular Materials and Engineering, 302(7), 1700002, (2017)

[22] Sasithorn, N., Martinová, L., Horáková, J., \& Mongkholrattanasit, R. Fabrication of Silk Fibroin Nanofibres by Needleless Electrospinning. Electrospinning: $\quad$ Material, Techniques, and Biomedical Applications, 95, (2016).

[23] Yalcinkaya, F., Yalcinkaya, B., \& Jirsak, O. Influence of salts on electrospinning of aqueous and nonaqueous polymer solutions. Journal of Nanomaterials, 2015, 1, (2015).

[24] Yener, F., Yalcinkaya, B., \& Jirsak, O. Roller Electrospinning System: A Novel Method to Producing Nanofibers, (2013).

[25] Niu, H., \& Lin, T. Fiber generators in needleless electrospinning. Journal of nanomaterials, 2012, 12, (2012). 